\title{
4-Labelings and Grid Embeddings of Plane Quadrangulations
}

\author{
Lali Barrière ${ }^{\star}$ and Clemens Huemer ${ }^{\star \star}$ \\ Departament de Matemàtica Aplicada IV, Universitat Politècnica de Catalunya \\ $\{$ lali, clemens\}@ma4.upc.edu
}

Finding aesthetic drawings of planar graphs is a main issue in graph drawing. Of special interest are rectangle of influence drawings. The graphs considered here are quadrangulations, that is, planar graphs all whose faces have degree four. We show that each quadrangulation on $n$ vertices has a closed rectangle of influence drawing on the $(n-2) \times(n-2)$ grid. Biedl, Bretscher and Meijer [2] proved that every planar graph on $n$ vertices without separating triangle has a closed rectangle of influence drawing on the $(n-1) \times(n-1)$ grid.Our method, which is completely different from that of [2], is in analogy to Schnyder's algorithm for embedding triangulations on an integer grid [9] and gives a simple algorithm.

Schnyder [9] showed that labeling the angles of a triangulation $T$ with 3 colors, with special rules, gives a 3-coloring and 2-orientation of the edges of $T$ such that the edges of each color form a directed tree. For each interior vertex of $T$, the three colored paths to the sinks of the respective trees divide $T$ into three regions. Counting the number of faces in each region gives the coordinates of the interior vertex in the grid drawing. Felsner [3] extended this result to the class of 3-connected plane graphs. In [8] it was studied to adapt this method to quadrangulations. In this case, the angles of a quadrangulation $Q$ can be colored with 2 colors, which gives an analogous 2-coloring and 2-orientation of the edges of $Q$ such that the edges of each color form a directed tree, and for each interior vertex the two colored paths to the respective sinks divide $Q$ into two regions. In [5] it is shown that counting the number of faces in a region of an interior vertex $v$ of $Q$ gives the coordinate of $v$ in a book embedding of $Q$ with two pages. Each page in this book embedding for $Q$ contains one of the two trees. Book embeddings of quadrangulations were also found in [6]. Whether this approach also gives a grid embedding for quadrangulations remained open.

We show here that labeling the angles of $Q$ with 4 colors instead of 2 (which gives a 4-coloring and 2-orientation of the edges) allows to obtain a pair of book embeddings of $Q$ such that the coordinates of a vertex $v$ in the two book embeddings are the coordinates of $v$ in the grid drawing of $Q$. It turns out that this embedding is a closed rectangle of influence drawing. It has the further property that edges of different colors are oriented in different directions (northeast, south-east, south-west, north-west). As a by-product of the rectangle of influence drawing, we also obtain a grid drawing of a quadrangulation on an

\footnotetext{
* Research supported by project MTM2008-06620-C03-01/MTM.

** Research supported by projects DGR 2009SGR-1040 and MEC MTM2009-07242.

D. Eppstein and E.R. Gansner (Eds.): GD 2009, LNCS 5849, pp. 413414 2010.

(C) Springer-Verlag Berlin Heidelberg 2010
} 
$\left\lceil\frac{n}{2}\right\rceil \times\left\lceil\frac{3 n}{4}\right\rceil$ grid by simple scaling. This is not optimal, because quadrangulations on $n$ vertices have a straight-line embedding on an $\left(\left\lceil\frac{n}{2}\right\rceil-1\right) \times\left\lfloor\frac{n}{2}\right\rfloor$ grid. However, the known algorithms by Biedl and Brandenburg [1] and Fusy [7], both require to add edges to make the quadrangulation 4-connected. An advantage of our simple algorithm is that it does not need to add edges and also works for quadrangulations with connectivity 2 .

Quadrangulations $Q$ are known to admit a touching segment representation: de Fraysseix, de Mendez and Pach [6] showed that one can assign vertical segments and horizontal segments to the vertices of $Q$ such that two segments touch if and only if the two corresponding vertices of $Q$ are adjacent. A different proof of this result, based on book embeddings of $Q$, is by Felsner et al. 4, who provided a bijection between the two trees of book embeddings of quadrangulations and rectangulations of a diagonal point set. The 4-labeling of a quadrangulation $Q$ gives two book embeddings and therefore two rectangulations by [4. This pair of rectangulations has the further nice property that in each rectangulation the boxes correspond isomorphically to the faces of $Q$ (that is, the dual graphs are isomorphic), both rectangulations have the same fixed outer face, and each segment intersects the line with slope 1 in one rectangulation and intersects the line with slope -1 in the other one.

This work builds upon previous results on binary labelings of quadrangulations from [58]. The novelty is the use of four colors instead of two and its application to the grid drawing. 4-labelings are in bijection with binary labelings from [5]. Using four colors allows us to get more insight into the combinatorial structure of quadrangulations.

\section{References}

1. Biedl, T., Brandenburg, F.: Drawing planar bipartite graphs with small area. In: Proceedings of the 17th Canadian Conference on Computational Geometry, Windsor, Canada, pp. 105-108 (2005)

2. Biedl, T., Bretscher, A., Meijer, H.: Rectangle of Influence Drawings of Graphs without Filled 3-Cycles. In: Kratochvíl, J. (ed.) GD 1999. LNCS, vol. 1731, pp. 359-368. Springer, Heidelberg (1999)

3. Felsner, S.: Convex Drawings of Planar Graphs and the Order Dimension of 3Polytopes. Order 18, 19-37 (2001)

4. Felsner, S., Fusy, E., Noy, M., Orden, D.: Bijections for Baxter Families and Related Objects (2008), http://arxiv.org/abs/0803.1546

5. Felsner, S., Huemer, C., Kappes, S., Orden, D.: Binary Labelings for Plane Quadrangulations and their Relatives (2008), http://arxiv.org/abs/math/0612021v3

6. de Fraysseix, H., de Mendez, P.O., Pach, J.: A left-first search algorithm for planar graphs. Discrete and Computational Geometry 13, 459-468 (1995)

7. Fusy, E.: Straight-line drawing of quadrangulations. In: Kaufmann, M., Wagner, D. (eds.) GD 2006. LNCS, vol. 4372, pp. 234-239. Springer, Heidelberg (2007)

8. Huemer, C., Kappes, S.: A binary labelling for plane Laman graphs and quadrangulations. In: Proceedings of the 22nd European Workshop on Computational Geometry, Delphi, Greece, pp. 83-86 (2006)

9. Schnyder, W.: Embedding Planar Graphs on the Grid. In: Proceedings of the first annual ACM-SIAM Symposium on Discrete Algorithms, pp. 138-148 (1990) 